\title{
L'Amour à deux visages: el agua en Pernette du Guillet como reflejo del diálogo amoroso
}

\section{Rodríguez-Navarro, María Victoria}

Universidad de Salamanca,

\begin{abstract}
Resumen
Sumergirse en el agua, reflejo simbólico de virtudes, es volver a las fuentes de la vida para regenerarse, para saciar esa sed de conocimiento y también para recibir los rayos del sol que en ella se reflejan. Éste es el caso de la lionesa Pernette du Guillet quien, a diferencia de Louise Labé, ha persistido en la memoria de los hombres no sólo por su talento natural sino gracias a la irradiación espiritual de la que la inundó Maurice Scève, «son Jour», jefe incuestionable del Círculo de Lyon, en el que las mujeres poetas del Renacimiento tuvieron un papel de primer orden. La mayoría de sus Rymes responden a una especie de diálogo amoroso entre ambos en el que el elemento líquido tiene un papel primordial. Pernette se presenta tan límpida como «l'eau de la claire fontaine», mientras que en el fondo anima a su poeta a todo lo contrario, produciéndose a veces un auténtico diálogo de sordos: ambos se aman con un amor sincero y mutuo pero no de la misma manera. Analizaremos estos aspectos confrontando los versos de Maurice Scève y los de Pernette du Guillet, en especial su Elegía II, para confirmar la oposición formal entre las ensoñaciones masculinas y femeninas.
\end{abstract}

Palabras clave: agua; amor; diálogo; conocimiento; purificación.

\begin{abstract}
Résumé
Se plonger dans l'eau, reflet symbolique de vertus, c'est revenir aux sources de la vie pour se régénérer, pour étancher une certaine soif de connaissance et pour recevoir encore les rayons du soleil qui se reflètent sur elle, comme c'est le cas de la lyonnaise Pernette du Guillet qui, à différence de Louise Labé, a persisté dans la mémoire des hommes non seulement à cause de son talent naturel, mais surtout au rayonnement spirituel dont Maurice Scève, son Jour, maître incontestable du Cercle de Lyon où les femmes poètes de la Renaissance ont eu un rôle primordial, l'a inondée. La presque totalité de ses «Rymes», répondent à une espèce de dialogue amoureux entre les deux où l'élément liquide joue un rôle déterminant. Pernette se présente aussi limpide que «l'eau de la claire fontaine», tandis qu'au fond encourage son poète à tout le contraire, se produisant de la sorte un authentique dialogue de sourds : les deux amis s'aiment d'un amour sincère et mutuel mais non de la même manière. Nous allons analyser ces aspects mettant en parallèle les vers de Maurice Scève et ceux de Pernette du Guillet, notamment l'Élégie II, pour confirmer l'opposition formelle entre les rêveries masculines et féminines.
\end{abstract}

Mots-clés: eau; amour; dialogue; connaissance; purification.

\begin{abstract}
Sinking in water, symbolic reflection of virtues, is like returning to the nurturing fountains of life to quench a thirst for knowledge and also to receive the sun rays reflected on it. This is the case of the attractive lady Pernette du Guillet who, unlike Louise Labé, has remained in men's memory not only for her natural talent, but thanks to the spiritual permeation given to her by Maurice Scève, «son Jour», unquestionable chief of the Lyon Circle. Here, poetesses of the Renaissance had a major role. Most of her Rymes are a kind of love dialogue between them, where the liquid element plays an essential part. Pernette presents herself as crystaline as «l'eau de la claire fontaine», but deep inside her, she leads her poet to the opposite, thus creating a real dialogue between deaf people: each one of them loves the other with a deep and sincere love, but not in the same way. I will analyze these aspects contrasting the poem by Maurie Scève and that by Pernette du Guillet, especially his Elegy II, with the aim of proving the formal opposition between male
\end{abstract}


and female daydreaming.

Keywords: water, love, dialogue, knowledge, purification.

Fuente, pozo, arroyo, río, lago, estanque, mar, océano, hielo, lluvia o niebla e incluso lágrimas. Desde los tiempos más antiguos el elemento líquido ha fascinado a los escritores y el simbolismo del agua aparece en infinidad de obras literarias sea cual sea la forma en la que se presente. Como dice Mathieu Castellani:

La mer offre le spectacle et l'aimable démesure. Stagnantes dans les marais, les eaux, image de la mort, exercent une autre séduction, non moins puissante. La douceur dormante des eaux tristes recèle les images de l'ennui. Par contraste les eaux claires, les eaux courantes, sont recherchées pour la rupture qu'elles instaurent au sein du précaire équilibre intérieur (Mathieu, 1975: 384).

El agua es pues polimórfica y ambivalente y por este motivo las referencias acuáticas pueden ser positivas y negativas según la función que se les quiera dar. Siempre en movimiento, cambiante, aparece como imagen del tiempo, como algo imposible de aprehender en su totalidad, y se escapa de manera irregular como la vida. Las aguas claras y transparentes aportan a la expresión del amor una tonalidad bien diferente, son, en palabras de Mathieu-Castellani «rêve de fraîcheur» ya que, cerca de ellas, «l'amant poursuit harmonieusement une rêverie apaisée, songe d'amour pur, comme l'onde transparent» (Mathieu, 1975: 392).

Su poder onírico, como señalaron Bachelard y Durand, hace que escribir sobre el agua, sobre cómo los poetas, novelistas o dramaturgos se han expresado apoyándose en ella, sea una especie de alquimia, resultado de mezclar el agua con la tinta según la bella expresión de Danièle Chauvin que recoge Cathérine Humières (Humières, 2007).

Si hay una mujer poeta cuya obra se pueda circunscribir al imaginario del agua ésa es Pernette du Guillet, quien, junto a Louise Labé, cierra el círculo de los poetas lioneses del Renacimiento. Mientras que Louise es fuego y pura pasión, Pernette es agua, ya sea cascada pura y cristalina, ya un tranquilo lago que refleja perfectamente la luz que irradia su gran maestro, Maurice Scève, su eterno enamorado, de cuya fuente de sabiduría bebió la joven poeta.

Aunque, según Schmidt su obra constituye uno de los hallazgos más preciosos de las letras femeninas (Schmidt, 1953: 227), Pernette no fue poeta por vocación sino que la poesía surgió en ella por la admiración que profesaba a Scève. Cuando se conocieron, él se encontraba en la cima de su gloria y la joven de 16 años se quedó impresionada: este momento le cambió la vida. Para el poeta fue un flechazo fulminante como escribió en el segundo dizain de su Délie: «Au premier oeil, mon Ame l'adora».

Pernette fue su gran amor de madurez, amor probablemente no consumado, siendo él para ella su «divin poète ». En sus encuentros, mientras cantaba y tocaba el laúd, Scève le leía versos « lui dévoilant les secrets de son art». Amistad pura en la dama que se transformó en él en un ardiente deseo; ella, como afirma Philippe Ford, la bien-aimée admirative (Ford, 2008:165), se dejaba querer, conduciendo un cierto sentimiento de exaltación amorosa a través del arte. En sus paseos campestres, sobre todo, asistimos a un diálogo amoroso en el que el agua va tener un papel primordial, pues, como en la vida misma, en palabras de Paul Ardouin, «tout ruisseau prend sa source en un point déterminé du globe, s'épanouit ensuite sous la forme d'une rivière, puis d'un fleuve qui se jette dans la mer. Il en est de même pour l'amour» (Ardouin, 1991: 14).

Lo que es realmente interesante es que sus conversaciones se pueden seguir a través de los dizains de Scève y de las respuestas de Pernette en sus Rymes como una correspondencia histórica. «À l'orage de ces disputes succèda le calme limpide des promenades champêtres» (Ardouin, 1991: 18). Salen, se pasean por las orillas de Ródano en las afueras de Lyon. De vez en cuando, el poeta reclama la «mercy» de la dama, que se enfada, pero al mismo tiempo se siente celosa y le reprocha que tenga otros amores. Como dice Ardouin «ainsi se poursuivent d'une manière oscillante et sinueuse, le dialogue d'amour des deux poètes» (Ardouin, 1991:18).

Le «divin poète» acompaña a su Délie, su gran amor, a quien adoraba por ser « de corps très belle et d'âme bellissime», como confiesa en su dizain 424. Su compenetración intelectual era tal que es bastante corriente encontrar el mismo tema 
en diferentes poemas creados por ambos. A modo de ejemplo, comparemos este poema de Pernette en el que ofrece una visión de la felicidad completa, una especie de sublimación del amor en una unión total de los amantes, rechazando todo lo que suena a gozo físico considerado como vulgar:

L’heur de mon mal, enflammant le désir,

Fit distiller deux cœurs en un devoir :

Dont l’un est vif pour le doux déplaisir,

Qui fait que Mort tient l'autre en son pouvoir.

Dieu aveuglé, tu nous a fait avoir

Du bien le mal en effet honorable :

Fais donc aussi que nous puissions avoir

En nos esprits contentement durable ! (Pernette: Ep XIII) ${ }^{1}$

con la décima de Scève donde recoge y transforma los términos empleados por su alumna. Le mal es denominado l’heur reafirmándose en su creencia de un bien deseable:

L'heur de nostre heur enflambant le desir

Unit double ame en un mesme povoir :

L'une mourant vit du doulx desplaisir,

Qui l'autre vive à fait mort recevoir.

Dieu aveuglé tu nous as fait avoir

Sans aultrement ensemble consentir,

Et posseder, sans nous en repentir,

Le bien du mal en effect désirable :

Fais que puissions aussi long temps sentir

Si doulx mourir en vie respirable. (Scève: 133$)^{2}$

Para W. J. A. Bots, le huitain 8 de Pernette et le dizain 133 de Maurice Scève s’appuient fortement sur ces combinaisons de mots, et leur comparaison détaillée s'avère doublement révélatrice et qu'un texte littéraire est souvent l'expression d'une expérience vécue et d'un parti pris d'écrivain» y añade que «Pernette et Scève se sont ingéniés à glisser dans leurs vers un message personnel, concret, faisant connaître leur attitude devant le sentiment naturel, mais accaparant qu'est l'amour illicite» (Bots,2005: 80). ${ }^{3}$ La admiración intelectual es mutua y ella se preocupa de dejárselo claro sobre todo al principio, cuando confiesa una atracción por su talento, sa vertu, sa grâce et sa faconde, más que por su belleza, lo que no impedía su gran éxito entre las mujeres, por eso no le duelen prendas al reconocer que se siente muy halagada:

Je suis tant bien que je ne le puis dire,

Ayant sondé son amitié profonde

Par sa vertu, qui à l'aimer m'attire

Plus que beauté : car sa grâce et faconde

Me font croire la première du monde. (Pernette.XVII)

\footnotetext{
${ }^{1}$ Pernette du Guillet: Rymes.

${ }^{2}$ Maurice Scève: Délie

${ }^{3}$ Propone dos interpretaciones sobre el tema basadas en los dos poemas:

Pernette: Comme mon amour non-physique (mon contentement spirituel) excite le désir, il oblige deux cœurs à le ressentir différemment, l’un vit pour (par) ce doux amour spirituel, l'autre (Scève) en est mort (n'en peut plus). Dieu cruel de l'amour, tu nous as fait goûter l'amour physique, en effet honorable ; fais donc aussi que nous en ayons un contentement spirituel durable.

Scève: $\quad$ Notre amour merveilleux excitant le désir, nous sommes en mesure de réunir nos âmes en un même acte d'amour : l'une languit par son doux amour insatisfait, ce qui fait que l'autre, plein d'ardeur, a possédé une morte. Dieu cruel de l'amour, tu nous as fait avoir, sans nous permettre de vivre ensemble, et posséder, sans que nous nous en repentions, le bien du mal (l'union des corps), en effet désirable; fais que nous puissions goûter encore longtemps ce doux mourir d'aimer en une vie supportable (respirable).
} 
Ve su intelecto recibiendo de manera privilegiada los rayos de la luz del autor de la Délie, al que llama Son Jour y reclama para ella el apelativo de la Journée, como si de este modo, cual superficie de agua transparente y cristalina, recibiera del sol la luz y el brillo de su maestro. Lo deja claro en la conocida Chanson IX. En realidad lo que Pernette hace aquí es una especie de paráfrasis del primer verso del dizain 376 de Scève: « u es le Corps, Dame, et je suis ton ombre».

\author{
Je suis la Journée, \\ Vous, Amy, le jour, \\ Qui m'a détournée \\ Du fâcheux séjour. \\ D’aimer la Nuit certes je ne veux point, \\ Pource qu'à vice elle vient toute à point : \\ Mais à vous toute être \\ Certes je veux bien, \\ Pource qu'en votre être \\ Ne gît que tout bien. \\ [...] \\ Ainsi éclairée \\ De si heureux jour, \\ Serai assurée \\ De plaisant séjour. (Pernette. IX)
}

Y en la misma línea:

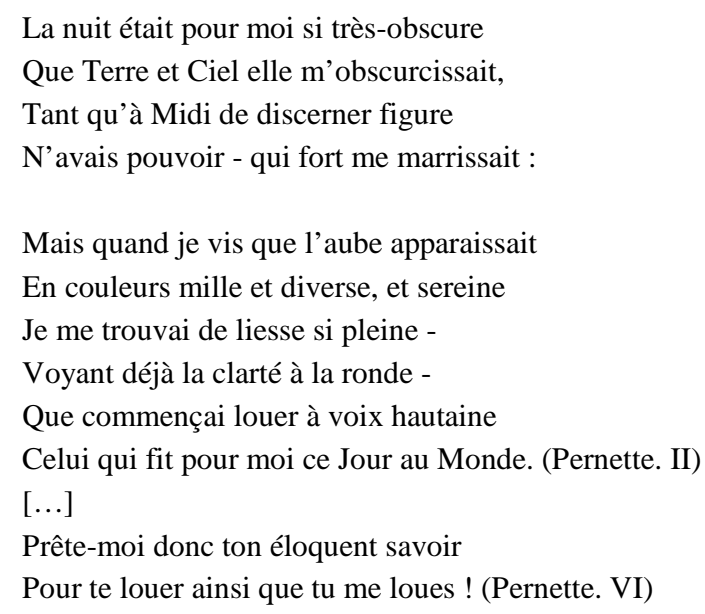

De este modo, en su epigrama IV lo compara a Apolo, «Esprit celeste et des Dieux transformé en corps mortel», con quien dice compartir «La vertu dont tu es la source et l'onde» sabiendo manejar como nadie «la plume fluante». Aquí vemos aparecer por primera vez el simbolismo del agua, como fuente de vida y de luz.

Esta especie de admiración casi mística explica lo esencial de su amor y de su obra. Leal consigo misma y con su situación, está más enamorada de su maître-poète, comparado unas veces con Júpiter, otras Apolo, que del hombre, quien le abre los ojos y le ilumina el intelecto y con quien puede establecer un diálogo de amor sublimado, como señala Bots «s'efforçant ainsi de lui faire partager, mais avec retenue, les joies corporelles et spirituelles que son pudique amour n’a pas manqué d’éveiller en elle» (Bots, 2005: 80). Así lo dice en sus famosos versos escritos en el epigrama XVI: 
L'âme et l'esprit sont pour le corps orner, Quand le vouloir de l'Eternel nous donne Sens et savoir pour pouvoir discerner

Le bien du bien que la raison ordonne.

Par quoi si Dieu de tels biens te guerdonne,

Il m'a donné raison qui a pouvoir

De bien juger ton heur et ton savoir.

Ne trouve donc chose si admirable,

$\mathrm{Si}$ à bon droit te désirent de voir

Le Corps, l’Esprit et l'Ame raisonnable. (Pernette, XVI)

Esta seducción intelectual no era suficiente para Scève, hombre acostumbrado a acumular conquistas femeninas, por más que ella le repitiera constantemente, con esa « parole chétive», término acuñado por Mathieu-Castellani (Mathieu, 1989), lo afortunada que se sentía por gozar de su amistad y todo lo que aprendía de su sabiduría y conocimiento:

Sais-tu pourquoi de te voir j'eus envie?

C'est pour aider à l'ouvrier, qui cessa,

Lors qu'assembla en me donnant la vie,

Les différents, où après me laissa.

Car m'ébauchant Nature s'efforça

D'entendre et voir pour nouvelle ordonnance

Ton haut savoir, qui m’accroît l'espérance

Des Cieux promise, ainsi que je me fonde,

Que me feras avoir la connaissance

De ton esprit, qui ébahit le Monde. (Pernette, XX)

Cuando él se queja de la situación reclamando un mayor acercamiento, la «mercy» tan deseada por su parte, se expresa en los términos siguientes, como se puede leer en esta décima:

A quoy pretendre yssir librement hors

D'une si doulce, \& plaisant servitude?

Veu que Nature \& en l'Ame, \& au Corps

En à jà fait, voire telle habitude,

Que plus tost veult toute solicitude,

Que liberté, loisir, \& leurs complisses.

Car en quictant Amour, \& ses delices,

Par Mort serois en ma joye surpris.

Parquoy enclos en si doubteuses lisses,

Captif je reste, \& sortant je suis pris. (Scève, 304)

Pernette intenta retenerlo de manera muy sutil prometiéndole más de lo que estaba dispuesta a darle, pero, con una pirueta de estilo, se excusa de no haber acudido a la cita y transforma su rechazo en otra nueva promesa en la que le asegura que está deseando ser suya, no un único día, sino para toda la vida:

Je te promis au soir que, pour ce jour,

Je m'en irais à ton instance grande

Faire chez toi quelque peu de séjour : 
Mais je ne puis... parquoi me recommande, Te promettant m'acquitter pour l'amende, Non d'un seul jour, mais de toute ma vie, Ayant toujours de te complaire envie.

Donc te supplie accepter le vouloir

De qui tu as la pensée ravie

Par tes vertus, ta grâce, et ton savoir. (Pernette, XIX)

Este poema, que para Ardouin es un modelo de diplomacia amorosa (Ardouin, 1991: 39), ofrece el retrato de una mujer coqueta que, en cierto modo juega con los sentimientos del poeta, porque no le importa repetir de nuevo:

Prenez le cas que, comme je suis vôtre -

Et être veux- vous soyez tout à moi :

Certainement par ce commun bien nôtre

Vous me devriez tel droit que je vous dois.

Et si Amour voulait rompre sa Loi,

Il ne pourrait l'un de nous dispenser,

S’il ne voulait contrevenir à soi,

Et vous, et moi, et les Dieux offenser. (Pernette, XXVI)

El momento en el que los dos puntos de vista están mejor confrontados se encuentra en el diálogo que llevan a cabo tras una cita en la que intercambiaron algunos de sus versos. En sus encuentros amistosos e intelectuales Scève no dejaba de implorar los favores a su dama, instalada en una especie de vaivén emocional como si fuera la superficie del agua pura y cristalina. En la décima 235 de Scève y en la famosa segunda elegía de Pernette es muy evidente que los dos poetas hacen referencia a la misma fuente que sería no sólo el escenario de un locus amenus sino la confidente de sus complicidades. El elemento líquido aparece aquí además como una ofrenda hídrica con una evidente función purificadora, capaz de apagar el deseo carnal del poeta para transformarlo y renovarlo.

Veamos y comparemos ambos. Para él, el agua es el receptáculo de la belleza del cuerpo de su amada, pudiendo purificar con su solo contacto cualquier tipo de líquido. Su rostro, sus ojos, sus labios son una ofrenda a la divinidad que el agua puede conservar en su interior para poder ser adorada como sagrada a partir de ese momento, como «un saint miroir»:

\author{
Aumoins toy, clere et heureuse fontaine, \\ Et Vous, ô eaux fraisches et argentines, \\ Quand celle en vous (de tout vice loingtaine) \\ Se vient laver ses deux mains yvoirines, \\ Ses deux Soleilz, ses levres corallines, \\ De Dieu creéz pour ce monde honnorer, \\ Debvriez garder pour plus vous decorer \\ L'image d'elle en vos liqueurs profondes. \\ Car plus souvent je viendroys adorer \\ Le sainct miroir de voz sacrées undes. (Scève, 235)
}

Como bien señala Bachelard, una sola gota de agua sirve para purificar todo un océano, como si de un bautismo se tratara. Estamos pues ante un agua viva, pura, transparente y tranquila, de tal modo que así se pueden entender esos ritos en los que se sumerge en el río a jóvenes vírgenes como un ejercicio para limpiar las aguas. Nuestra dama entra en el agua desnuda, con una desnudez que simboliza la pureza y ella y el líquido se purifican mutuamente; sin embargo, este momento virginal es frágil y a menudo está amenazado por una mirada insolente como aparece en la elegía de Pernette en la que presenta el momento sous le couvert de un sueño, alejando así por pudor cualquier intención personal al referirlo 
en primera persona. La dama vive esta ensoñación del otro lado del espejo y si hace el regalo de su desnudez es porque se siente protegida por la presencia imaginaria de sus hermanas las ninfas. Aquí ya no se habla sólo de agua purificadora sino de «eau amoureuse»:

\author{
Combien de fois ay-je en moi souhaieté \\ Me rencontrer sur la chaleur d'esté \\ Tout au plus pres de la clere fontaine, \\ Où mon desir avec cil se pourmaine \\ Qui exercite en sa philosophie \\ Son gent esprit, duquel tant je me fie \\ Que ne craindrois, sans aucune maignie, \\ De me trouver seule en sa compaignie : \\ Que dy-je : seule ? ains bien accompaignée \\ D’honnesteté, que Vertu a gaignée \\ A Apollo, Muses, et Nymphes maintes, \\ Ne s'adonnantz qu’à toutes œuvres sainctes.
}

La elegía, muy rica en alusiones mitológicas, comienza con el motivo central de la femme au bain - la claire fontaine, la femme nue au bain- y se enriquece con la presencia de Apolo, Scève sin duda, y las musas y ninfas que la acompañan, para remitirnos enseguida al episodio del baño de Diana, interrumpido por Actéon. Los paralelismos entre la diosa y Pernette son más que evidentes. Comparten el rigor extremo de su virtud y ambas se presentan como la virgen insumisa, indomable como el agua, insensible, cruel e incluso violenta. Pernette no se somete físicamente a los deseos del hombre y sin embargo parece insensible al sufrimiento de su poeta. Triunfa sobre el amor carnal para entregarse exclusivamente al amor intelectual. Sin embargo es obvio que su extrema coquetería le lleva a la provocación, dejándole acercarse con atrevimiento para enseguida alejarlo lanzándole a los ojos agua que limpie una mirada más o menos sucia:
L'a quand j'aurois bien au long veu son cours,
Je le lairrois faire appart ses discours :
Puis peu à peu de luy m'escarterois,
Et toute nue en l'eau me gecterois :
Mais je vouldrois lors quant, et quant avoir
Mon petit Luth accordé au debvoir,
Duquel ayant congneu, et pris le son,
J'entonnerois sur luy une chanson
Pour un peu veoir quelz gestes il tiendroit :
Mais si vers moy il s'en venoit tout droict,
Je le lairrois hardyment approcher :
Et s'il vouloit, tant soit peu, me toucher,
Lui gecterois (pour le moins) ma main pleine
De la pure eau de la clere fontaine,
Lui gectant droict aux yeulx, ou à la face.

Canta para él con su laúd para atraerlo y después castigarlo ${ }^{4}$ pero no pretende ahogar a Scève en el agua sino en la pasión y en la desesperación, en una actitud mucho peor intencionada que la de las ninfas, testigos mudos en esta escena. Hay una diferencia entre la diosa de la caza y ella: Diana es sorprendida en el baño y Pernette provoca esta situación deliberadamente pues como afirma Ford, « elle sait à l'avance les effets que ces actions exerceront sur l'amant» (Ford: 172) pretendiendo sin embargo recrear en este momento el episodio de Actéon y sentirse una diosa.

\footnotetext{
${ }^{4}$ En la décima 345 de la Délie, Scève recrea este momento del laúd cuando compara el abrazo al instrumento musical con el que desearía a su persona: Entre ses bras, ô heureux, près du coeur / Elle te serre en grand'delicatesse.
} 


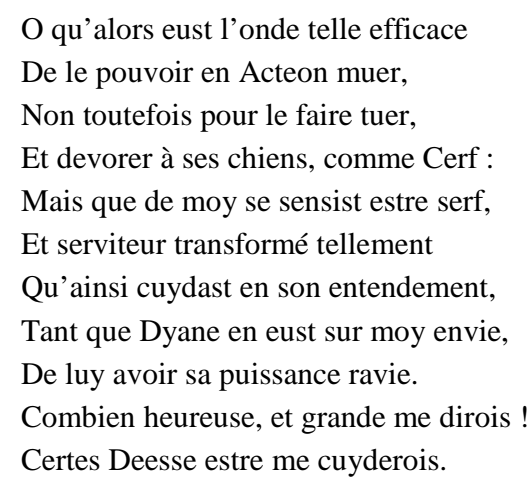

En el mito, Actéon se metamorfosea en ciervo y es devorado por sus propios perros. Es bien sabido que en el siglo XVI el perro estaba considerado como un animal brutal. Aparece allí donde se quiere hablar de desmesura, especialmente en lo referente al deseo sexual. Simboliza pues el castigo ejemplar: los perros vengan la pureza de la imagen desnuda. Acteón sufre el suplicio que quiere hacer sufrir a Diana. Sin embargo, en el poema, la acción del agua ayuda a enmendar el pecado por esa función purificadora antes mencionada y no sólo limpia su mirada sucia sino que esta agua fresca tiene el poder de renovación y resurrección del poeta: Pernette quiere transformar a su muy humano enamorado en un ser sometido a ella, que pase de «cerf» a «serf». Ya sea en el mito o en el poema, el hombre es siempre la víctima.

Sin embargo, al final parece recapacitar y creyéndose más inteligente que Diana, le salva la vida para, de este modo, tenerlo para siempre como esclavo. Se cuida mucho de disgustar a los dioses y a las musas, privándoles de un poeta tan brillante como Scève, tan cerca de la divinidad en el neoplatonismo. Y en un ejercicio, otra pirueta más, de estilo, lo ensalza exageradamente menospreciándose ella misma.

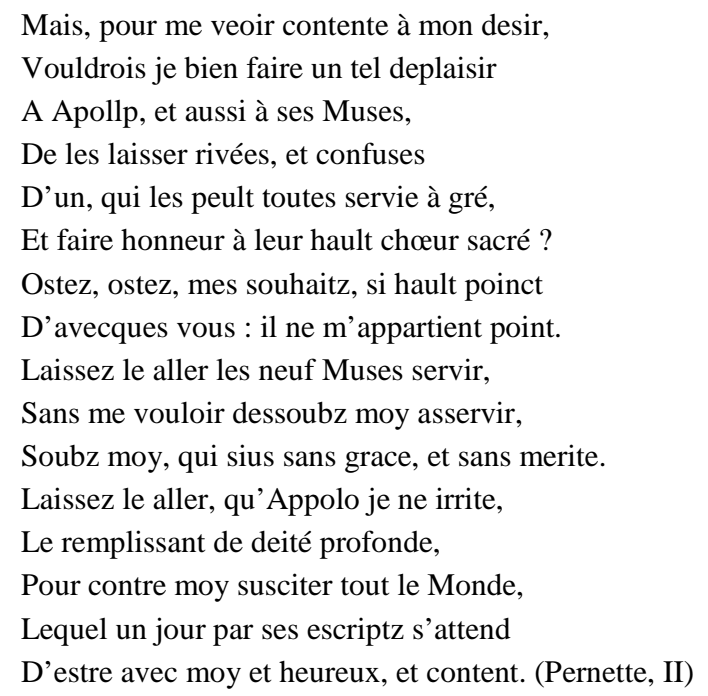

A través de este espejismo o ensoñación comprobamos perfectamente cómo Pernette aspiraba por encima de todo a la unión de las almas, lo que era el caso de Scève pero pretendiendo éste algo más. La diferencia entre ambos poemas es más que evidente, como lo es entre sus dos maneras de mostrar el amor. En sus Rymes, ella, aunque alaba constantemente el talento y la «vertu» de su amante, se queja de la persistencia de sus apetencias sensuales, mientras que el autor de la Délie, a pesar de que loa la divina pureza de la dama, la acusa de no hacer caso a sus deseos. Se trata de un diálogo de sordos, pues se aman sinceramente pero de diferente manera. Para Tristan Vigliano, esta relación, opinión que comparto, se puede resumir en «un désir réciproque, librement partagé, et qui place les deux amants dans un rapport d’égalité : la poétesse, pour avoir désiré le savoir de son aimé, finit par se l'approprier et entend ainsi faire, à son tour, l’objet du même 
désir qu'elle a la première éprouvé» (Vigliano, 2007). Y ambos poemas reflejan el contraste entre las ensoñaciones masculinas y femeninas en el cuadro emblemático de «l'eau amoureuse».

Pernette busca en el conocimiento no sólo la ciencia sino también una fuente de luz y por consiguiente un elemento de purificación. Como afirma Ardouin, «les révelations de la science sont comparables au scintillement lumineux de l’eau caressée par les rayons de soleil» para continuar diciendo que para Pernette, su estado de ánimo es comparable a la « fluidité de l'eau qui coule, mais c’est Maurice Scève qui est chargé d’opérer la purification» (Ardouin, 1991: 29). Toda su obra responde a las características y a los valores del agua dulce, la de los ríos, los lagos y las fuentes. E incluso, podría vislumbrarse otro símbolo del agua, el agua inmóvil como espejo inalterable de la poeta, ya que a veces su amor parece intemporal, como si se tratara de un presente eterno y permanente; así podría interpretarse en uno de sus versos, concretamente el epigrama XVI, cuando afirma respecto a la muerte, en una idea totalmente neoplatónica, que « ce mourir engendre une autre vie».

Si, parafraseando a Jean-Pierre Richard, amar a alguien es adivinar en esa persona la frescura que le queda de su infancia y buscar en él el agua de la vida, habría que concluir señalando que el agua cumple un papel primordial en el amor, ya que el hombre busca en el agua el elemento más femenino y más uniforme de la naturaleza. Como a Pernette, el agua nos hace soñar, ya sea agua del mar, del rio, de un pozo, de la misma lluvia; nos purifica, nos renueva y nos aporta la salvación y la vida. Y nunca está en contradicción con la pasión amorosa, más bien es una asociación paradójica, pues según Mathieu- Castellani, «l'eau ne tue pas la flamme amoureuse, elle est liée à l'amour heureux. Sa pureté et transparence font que l’amant se plaît auprès d'elles» (Mathieu, 1975: 395).

\section{Referencias bibliográficas}

Ardouin, Paul (1991). Pernette du Guillet : l'heureuse Renaissance, miracle de l'amour, de la lumière et de la poésie. Paris : Nizet.

Ardouin, Paul (1981). MAurice Scève, Pernette du Guillet, Louise Labé : l'amour à Lyon au temps de la Renaissance. Paris : Nizet

Bots, Wim J. A. (2005). Pernette du Guillet (1520-1545), Amour humaniste ? Amour humain ? en Bulletin de l'Académie du Var, pp.

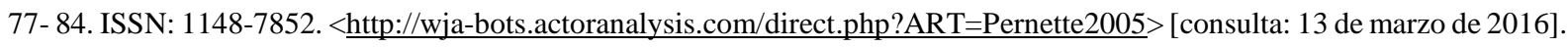

Du Guillet, Pernette (1981). Rymes, édition de Victor Graham. Genève Droz.

FORD, Philippe (2008). «À propos de quelques élégies de Pernette du Guillet» en L'émergence littéraire des femmes à Lyon à la Renaissance 1520-1560. Publications de l’université de Saint-Etienne. Nouvelles recherches n³. pp. 165-76.

Humieres, Catherine (2007). «Écrire sur l'eau», en Acta fabula, vol. 8, $\mathrm{n}^{\circ} \quad 3, \quad$ Mai-Juin 2007, $<$ http://www.fabula.org/acta/document3370.php> [consulta: 20 de marzo de 2016].

Mathieu-Castellani, Giselle.(1989). «La parole chétive: les Rymes de Pernette du Guillet» en Littérature, n73 « Mutations

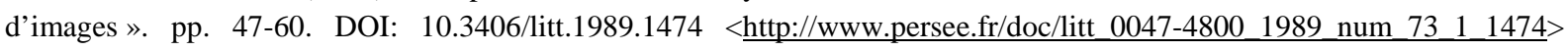
[consulta: 5 de abril de 2016].

Mathieu-Castellani, Giselle. (1975). Les thèmes amoureux dans la poésie française (1570-1600), Paris: Klincksieck.

SCEVe, MAurice (1985). Délie, objet de plus haute vertu, édition de Françoise Charpentier. Paris: Gallimard.

SchmidT, Albert-Marie (1953). Poètes du XVI siècle. Bibl. De la Pléiade (notice pp. 227-228).

SchMIDT, Albert-Marie (1967). Études sur le XVI siècle. Paris: Albin Michel.

Vigliano, Tristan (200. «Pernette en ses rymes» en Acta fabula, vol. 8, nº 1, Janvier-février 2007,

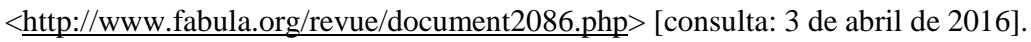

